\title{
Dank an die Gutachter
}

Herausgeber und Schriftleitung danken allen Gutachterinnen und Gutachtern herzlich, die durch ihre Unterstützung im vergangenen Jahr maßgeblich zur Qualität von Forschende KomPLEMENTÄRMEDIZIN beigetragen haben:

Frank Andersohn, Berlin Marcus Bäcker, Essen

Tamás Bender, Budapest

Tom Bernatik, Erlangen

Marcus Bernhörster, Frankfurt/M.

Shona Blair, Sydney

Michael Boschmann, Berlin

Thomas Brockow, Bad Elster

Arndt Büssing, Herdecke

Gustav J. Dobos, Essen

Friedrich Edelhäuser, Herdecke

Reiner Eichenberger, Fribourg

Axel Eustachi, München

Albrecht Falkenbach, Bad Ischl

Torkel Falkenberg, Huddinge

Matthias Fink, Hannover

Gerd Fricker, Heidelberg

Ingrid Gerhard, Heidelberg

Gerd Glaeske, Bremen

Corina Güthlin, Frankfurt/M.

Tibor Hajto, Budapest

Harald J. Hamre, Freiburg i.Br.

Andreas Hanses, Dresden

Peter Heusser, Herdecke

Markus Horneber, Nürnberg

Katarina Hostanska, Zürich

Roman Huber, Freiburg i.Br.

Jutta Hübner, Frankfurt/M.

Josef Hummelsberger, München

Hubert Janik, Rostock
A. Jaya Chitra, Madurai

Helle Johannessen, Odense

Stefanie Joos, Heidelberg

Robert Jütte, Stuttgart

Arturas Kazemekaitis, Kaunas

Christian Kessler, Hannover

Curt Kösters, Hamburg

Karin Kraft, Rostock

Nils Krähmer, Ottobeuren

Hans Christoph Kreck, Frankfurt/M.

Ponnusamy Kumar, Grand Cayman

Jost Langhorst, Essen

George Lewith, London

Klaus Linde, München

Gerhard Litscher, Graz

Rainer Lüdtke, Essen

Clare McDermott, Southampton

Remy Meier, Liestal

Karin Meissner, München

Jörg Melzer, Zürich

Andreas Michalsen, Berlin

Susanne Moebus, Essen

Antonio Morandi, Mailand

Karsten Münstedt, Gießen

Frauke Musial, Essen

Arya Nielsen, New York, NY

Menachem Oberbaum, Jerusalem

Martin Offenbächer, Schondorf

Miriam Ortiz, Berlin

Thomas Ostermann, Herdecke
Daniela Paepke, München

Florian Pfab, Charlestown, MA

Thomas Rampp, Essen

Jürgen Reichling, Heidelberg

Matthias Rostock, Zürich

Christoph Schempp, Freiburg i.Br.

Rolf Schloesser, Frankfurt/M.

Leslie Schrage-Leitner, Wien

Hartmut Schröder, Frankfurt/O.

Florian Schwerla, Gauting

Sarangapani S. Sreelatha, Singapore

Rainer Stange, Berlin

Wolfram Stör, Icking

Christian Terreaux, Villars-sur-Glane

Gerhard Tucek, Krems

Bernhard Uehleke, Zürich

Gudrun Ulrich-Merzenich, Bonn

Robbert van Haselen, Baden Baden

Cornelia von Hagens, Heidelberg

Harald Walach, Frankfurt/O.

Carolin S. Weckerle, Zürich

Wolfgang Weidenhammer, München

Joachim Weis, Freiburg i.Br.

Axel Wiebrecht, Berlin

Francoise Wilhelmi de Toledo, Überlingen

Matthias Wischner, Stade

Ursula Wolf, Bern

Garret Yount, San Francisco, CA

Renatus Ziegler, Arlesheim

\section{KARGER}

Fax +497614520714

Information@Karger.de

www.karger.com 